\title{
Correction to: Changing epidemiology of hemorrhagic fever with renal syndrome in Jiangsu Province, China, 1963-2017
}

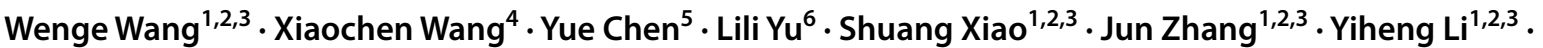 \\ Shangzhi Hong ${ }^{1,2,3} \cdot$ Liqun Fang $^{7} \cdot \operatorname{Jian} \mathrm{Hu}^{1,2,3} \cdot \mathrm{Yi} \mathrm{Hu}^{1,2,3} \cdot$ Yuanfang Qin ${ }^{4} \cdot$ Ming Yue $^{8} \cdot$ Yun Zhang ${ }^{9} \cdot$ Bin Zhang $^{9}$. \\ Jianli Hü $\cdot$ Zhijie Zhang ${ }^{1,2,3}$
}

Published online: 17 February 2022

○) Springer-Verlag GmbH Germany, part of Springer Nature 2022

\section{Correction to: Journal of Public Health: From Theory to Practice https://doi.org/10.1007/s10389-021-01526-w}

2. The corrected Fig. 2 is given below.

Wenge Wang and Xiaochen Wang contributed equally to this article and share first authorship.

The original article can be found online at https://doi.org/10.1007/ s10389-021-01526-w.

Jianli $\mathrm{Hu}$

jshjl@jscdc.cn

$\triangle$ Zhijie Zhang epistat@gmail.com

1 Department of Epidemiology and Biostatistics, School of Public Health, Fudan University, Shanghai 200032, China

2 Key Laboratory of Public Health Safety, Ministry of Education, Shanghai 200032, China

3 Laboratory for Spatial Analysis and Modeling, School of Public Health, Fudan University, Shanghai 200032, China

4 Department of Acute Infectious Disease Control and Prevention, Jiangsu Provincial Center for Disease Control and Prevention, Nanjing 210009, Jiangsu, China

5 Department of Epidemiology and Community Medicine, Faculty of Medicine, University of Ottawa, Ottawa, Ontario K1H 8M5, Canada
6 Peace Center for Biostatistics, Jiann-Ping Hsu College of Public Health, Georgia Southern University, Statesboro, GA 30460, USA

7 State Key Laboratory of Pathogen and Biosecurity, Beijing Institute of Microbiology and Epidemiology, Beijing 100071, China

8 Department of Infectious Diseases, The First Affiliated Hospital of Nanjing Medical University, Nanjing 210029, Jiangsu, China

9 Institute of Epidemiology and Microbiology, Eastern Theater Command Centers for Disease Control and Prevention, Nanjing 210002, Jiangsu, China 
The original article has been corrected.
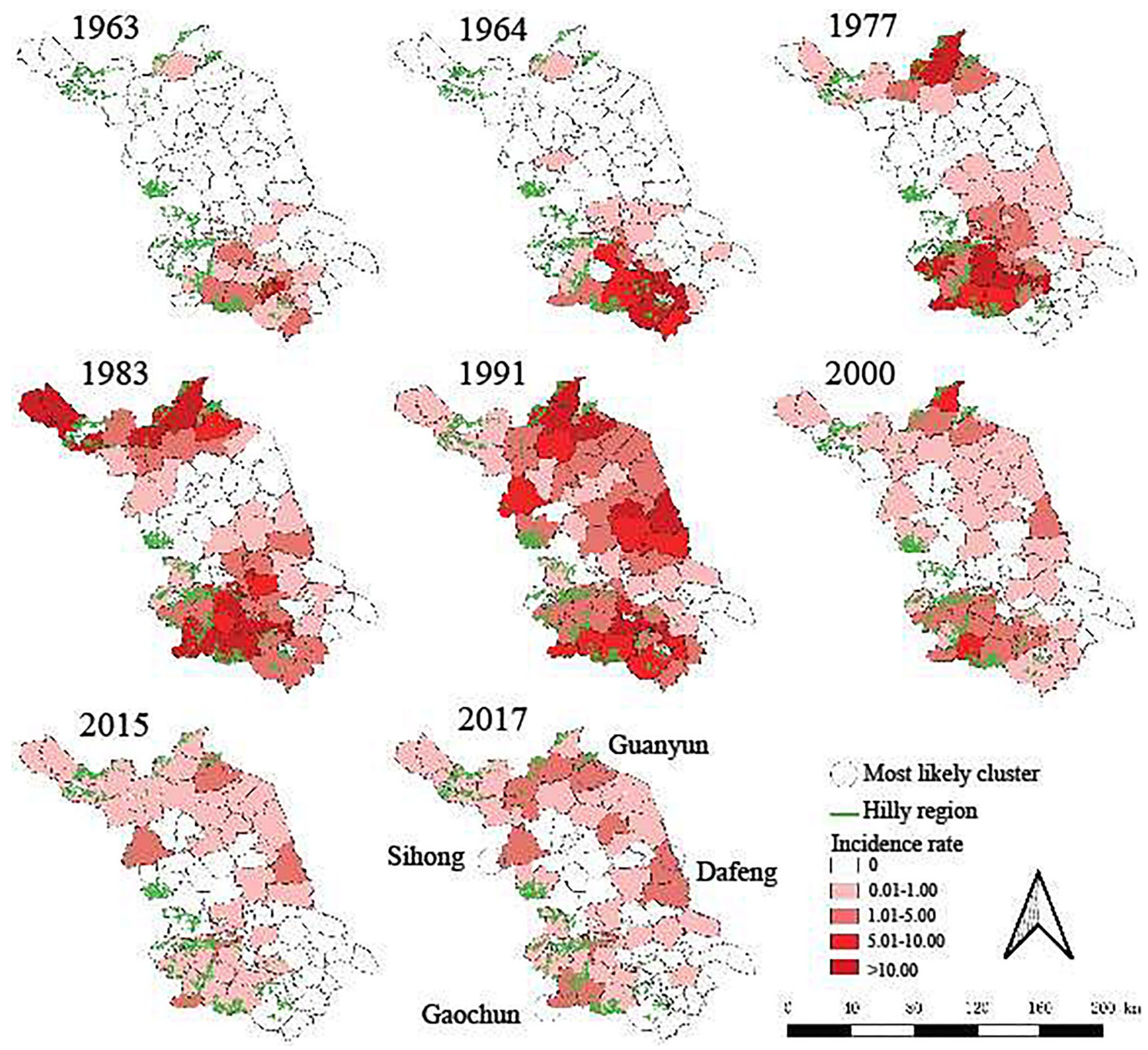

Most likely cluster

- Hilly region Incidence rate

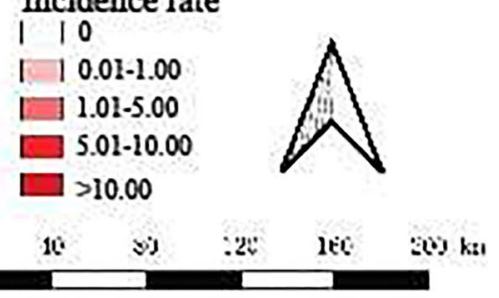

Publisher's note Springer Nature remains neutral with regard to jurisdictional claims in published maps and institutional affiliations. 